\title{
ON ANALYTIC IRREDUCIBILITY AT $\infty$ OF A PENCIL OF CURVES
}

\author{
T. T. $\mathrm{MOH}$
}

\begin{abstract}
In this article we establish that if a member of the pencil $f(x, y)+\lambda$. is analytic irreducible at $\infty$ then all members are.
\end{abstract}

1. Introduction. In [3] W. Engel used the following statement: "For a special member of the pencil $f(x, y)+\pi=0$ the number of branches at $\infty$ cannot be greater than the corresponding number for the general one". The above statement has been a main blockade for understanding his proof of the theorem of integral Cremona-transformation or the global Jacobian theorem. In $1971 \mathrm{~S}$. S. Abhyankar disproved it by giving a counterexample (unpublished). However a weaker statement (see Theorem I) can be proved easily by applying the results of [4]. The notations and results of [1], [2], [4] will be used extensively. We shall assume that the ground field $k$ is algebraically closed with characteristic zero.

2. Theorem I. Let $f(x, y)$ be a polynomial of degree $n$ defining a curve with only one place at $\infty$ centered at the infinite point on the $x$-axis. Let $\left(\mu_{i}\right)$ be defined as in [1], [2], [4]. Let $d=\max _{i} \mu_{i} / d_{i} n$. Note that $d$ is a positive number.

THEOREM I. The curve defined by $f(x, y)+p(x)$ with $\operatorname{deg} p(x)<d$ has only one place at $\infty$. In particular, $f(x, y)+c$ is analytically irreducible at $\infty$ for arbitrary constant $c$ in $k$.

Proof. Since $f(x, y)$ defines a curve with one place at $\infty$, then it follows from Theorem 1 of [4] that there is an ordered factorization of $n=n_{1}, n_{2}, \cdots, n_{h}$ such that the $r$ th power conditions are satisfied $\forall r \leqq h$. Let us use this factorization for $f(x, y)+p(x)$ also. Let $g_{r}(x, y)$ be the approximate $d_{r}$ th root of $f(x, y)$, namely $g_{r}(x, y)$ is the unique polynomial with

$$
\operatorname{deg}_{y}\left(f(x, y)-g_{r}(x, y)^{d r}\right)<n-\left(n / d_{r}\right) .
$$

Received by the editors May 29, 1973 and, in revised form, August 30, 1973.

AMS (MOS) subject classifications (1970). Primary 14C20.

Key words and phrases. Analytic irreducibility, approximate $d$ th root of a polynomial, place.

(c) American Mathematical Society 1974 
Clearly

$$
\operatorname{deg}_{y}\left(f(x, y)+p(x)-g_{r}(x, y)^{d r}\right)<n-\left(n / d_{r}\right) .
$$

In other words $g_{r}(x, y)$ is the approximate $d_{r}$ th root of $f(x, y)+p(x)$. Let us verify the conditions (1), (2) and (3) in Definition 4.2 of [4] for $f(x, y)+p(x)$ and the ordered factorization $n=n_{1}, n_{2}, \cdots, n_{h}$. Let

Clearly

$$
f(x, y)=g_{r}(x, y)^{d_{r}}+\sum_{i=1}^{d_{r}}\left(\sum_{\alpha} a_{i \alpha} g^{\alpha}\right) g_{r}(x, y)^{d_{r-i}}
$$

$$
f(x, y)+p(x)=g_{r}(x, y)^{d r}+\sum_{i=1}^{d r}\left(\sum_{\alpha} a_{i \alpha} g^{\alpha}\right) g_{r}(x, y)^{d_{r-i}}+p(x) .
$$

The corresponding polynomials in Definition 4.2 of [4] for $f(x, y)$ and $f(x, y)+p(x)$, respectively, are as follows

$$
y^{d r}+\sum_{i=1}^{d r}\left(\sum a_{i \alpha} x^{\alpha}\right) y^{d r-i}=\sum f_{i}\left(x_{1}, \cdots, x_{n}\right) y^{d r-i},
$$

and

$$
y^{d_{r}}+\sum_{i=1}^{d_{r}}\left(\sum a_{i \alpha} x^{\alpha}\right) y^{d_{r-i}}+p(x)=\sum f_{i}\left(x_{1}, \cdots, x_{n}\right) y^{d_{r-i}}+p(x) .
$$

According to the $r$ th power condition for $f(x, y), \mu_{r}=d_{r}\left|\sigma_{r} f_{d_{r}}\left(x_{1}, \cdots, x_{n}\right)\right|$ is strictly bigger than $\left|\sigma_{r} p(x)\right|=n \operatorname{deg} p(x)$ by our restriction on the degree of $p(x)$. It trivially follows that conditions (1), (2) and (3) in Definition 4.2 in [4] are satisfied. Our theorem follows from Theorem I of [4]. Q.E.D.

Let $f(x, y)$ be a polynomial defined by a rational curve with one place at $\infty$; it follows from Theorem I that $f(x, y)+\lambda$ will define the curve with one place at infinity. We propose

Conjecture. Let $f(x, y)$ be as previous. The polynomial $f(x, y)+\lambda$ defines rational curves for some nonzero $\lambda$ iff $f(x, y)$ defines a nonsingular rational curve.

Remark. After this manuscript had been prepared S. S. Abhyankar showed the author another proof by applying (3.4) of [1]. In fact, as pointed out by him, the condition "only one place at $\infty$ " can be replaced by "only one place at point $p$ " without changing either proof.

\section{REFERENCES}

1. S. S. Abhyankar and T. T. Moh, Newton-Puiseux expansion and generalized Tschirnhausen transformation. I, Crélle 260 (1973), 47-83. 
2. S. S. Abhyankar and T. T. Moh, Newton-Puiseux expansion and generalized Tschirnhausen transformation. II, Crélle 261 (1973), 29-54.

3. W. Engel, Ein Satz über ganze Cremona-Transformationen der Ebene, Math. Ann. 130 (1955), 11-19. MR 17, 787.

4. T. T. Moh, On approximate roots of a polynomial, Crélle (to appear).

Department of Mathematics, University of Minnesota, Minneapolis, MinneSOTA 55455 